Eco matemático ISSN 1794-8321 E-ISSN 2462-8794 Volumen 8 (s1) 2017, páginas 68 - 72

\title{
Contaminantes emitidos por los hornos colmena en empresas Cerámicas del Municipio del Zulia, Norte de Santander a través de la modelización de concentraciones
}

\section{Contaminating emits for the hive ovensceramic companiesof municipality of the Zulia, Norte de Santander throughof the modeling of gathering}

\author{
Contaminação de emissões para as empresas coletoras de ovário do município de Zulia, Norte de \\ Santander, através da modelagem de coleta \\ Sonia Maritza Mendoza- Lizcano, ${ }^{\mathrm{a} *}$ Cesar Orlando Vargas- Mantilla ${ }^{\mathrm{b}}$ \\ ${ }^{* a}$ Doctora en Educación - Universidad Pedagógica Experimental Libertador. (10 \\ ${ }^{b}$ Tecnólogo Químico - Universidad Francisco de Paula Santander. (1)
}

Forma de citar: Mendoza, S., Vargas, C. (2016). Contaminantes emitidos por los hornos colmena en empresas Cerámicas del Municipio del Zulia, Norte de Santander a través de la modelización de concentraciones. Encuentro Internacional en Educación Matemática Versión ISSN 2539-1885. La Educación Matemática como Herramienta en el Desempeño Profesional Docente. Cúcuta, Colombia. 68 - 72.

\begin{abstract}
Resumen: El sector de la arcilla es referente en la industria del Norte de Santander, con 87 empresas legalmente establecidas y una participación del 12,8\% de Producto Interno Bruto (PIB). Elaboran productos en arcilla, para el sector de la construcción; como tabletas, ladrillos, tejas, bloques, entre otros, comercializados nacional o internacionalmente. En el proceso productivo, los hornos colmena utilizados emiten contaminantes a la atmósfera a través de las chimeneas. El Ministerio de Ambiente, Vivienda y Desarrollo Territorial estableció normas y estándares de emisión admisibles de contaminantes a la atmósfera para este tipo de industrias, así como el seguimiento y controla las fuentes fijas contaminantes con periodicidad de un año o menos si los resultados lo ameritan. Estos análisis son costosos y los realizan laboratorios especializados ubicados fuera del departamento. Se considera implementar métodos alternativos de control, a través de la formulación de un modelo matemático que estime las concentraciones de contaminantes emitidos por estos hornos en la fabricación de productos cerámicos del sector del Zulia Norte de Santander. Desarrollando una investigación cuantitativa, basada en la identificación y cuantificación de variables incidentes en el proceso de cocción y el análisis estadístico de históricos de los estudios de evaluación de emisiones atmosféricas en fuentes fijas, para establecer patrones de comportamiento; que a su vez se puede convertir en una herramienta de seguimiento y control continuo de contaminantes de los procesos de cocción desarrollados; situación que apunta a un conocimiento pertinente de los procesos y responsabilidades ante los estamentos nacionales.
\end{abstract}

Palabras clave Contaminación, Chimenea, Emisiones, Hornos colmena, Isocinéticos, Modelos

\begin{abstract}
The sector of the clay is regarding in the Industry of Norte de Santander, with 87 companies established legally establisheds and an involvement of the $12,8 \%$ of gross internal product (PIB). This produced products in clay, for the sector of the construction; like tablets, bricks, tiles, blocks, among others, commercializes national or internationally. In the productive process, the hive ovens use to emit contaminating's to the atmosphere through of the chimneys, the ministry of environment, home and territorial development this established regulations and standards of transmission admissible of contaminating to the atmosphere for this kind of industries, as well as the monitoring and control to the permanent sources contaminating with periodicity of one year or less if the results the merit.These analysis are expensive and the this makes laboratories specialized located out of the state. Is considers implement methods alternatives of control, through of the formulation of a model mathematical that respect to the gathering of contaminating emits for this ovens in the manufacturing of ceramics products of the sector of Zulia from Norte de Santander. Developing an investigation quantitative, this is based in the identification and quantification of variables incidental in the process of boiling and the analysis statistical of historical of the studies of evaluation of transmissions atmospherics in permanent sources, for establish patterns of performance; what at the same time is can transform in an analytic tool of monitoring and control constant of contaminating of the process of boiling develops; Situation that indicate to an knowledge relevant of the process and responsibilities facing the stratum nationals.
\end{abstract}

Keywords Contamination, Chimney, Hive ovens, Isokinetic, Models, Oven.

* Autor para correspondencia soniamaritza@ufps.edu.co

2590-9215@ 2017 Universidad Francisco de Paula Santander. Este es un artículo bajo la licencia CCBY 
Resumo: O setor da argila está em causa na Indústria do Norte de Santander, com 87 empresas estabelecidas legalmente estabelecidas e um envolvimento dos $12,8 \%$ do produto interno bruto (PIB). Isso produzia produtos em argila, para o setor da construção; como tablets, tijolos, telhas, blocos, entre outros, comercializa nacional ou internacionalmente. No processo produtivo, os fornos das colméias costumam emitir contaminantes para a atmosfera através das chaminés, do ministério do meio ambiente, do território e do desenvolvimento territorial, estabelecendo normas e padrões de transmissão admissíveis de contaminação para a atmosfera por esse tipo de indústrias, bem como como o monitoramento e controle das fontes permanentes contaminando com periodicidade de um ano ou menos se os resultados obtiverem o mérito. Essas análises são caras e isso torna os laboratórios especializados localizados fora do estado. Considera-se implementar métodos alternativos de controle, através da formulação de um modelo matemático que respeite ao recolhimento de emissores contaminantes para esses fornos na fabricação de produtos cerâmicos do setor de Zulia, do Norte de Santander. Desenvolvendo uma investigação quantitativa, esta baseia-se na identificação e quantificação de variáveis incidentais no processo de ebulição e a análise estatística de histórico dos estudos de avaliação de transmissões atmosféricas em fontes permanentes, por estabelecer padrões de desempenho; o que ao mesmo tempo é pode transformar em uma ferramenta analítica de monitoramento e controle constante de contaminação do processo de ebulição desenvolve; Situação que indica um conhecimento relevante do processo e responsabilidades frente aos estratos nacionais.

\section{Palavras chave Contaminação, Chaminé, Fornos de Colmeia, Isocinética, Modelos, Forno.}

\section{Introducción}

Según (Díaz, 2013), la responsabilidad empresarial con la sociedad y la naturaleza se da cuando ésta se responsable de encarga de los residuos que genera tanto en los procesos productivos como durante la vida útil del producto mismo. Por otra parte Sarabia, Sánchez \& Leiva, (2017), señalan que en Colombia el sector de la construcción es uno de los mayor crecimiento económico y generador de grandes cantidades de residuos producto de la demolición, o la fabricación de los materiales de construcción. Considerando que "los municipios de Cúcuta, Los Patios, Villa del Rosario, El Zulia y San Cayetano, representan el $10 \%$ de la extensión del departamento de Norte de Santander, y el 6,85\% de su territorio lo ocupa la industria cerámica“ ( Sánchez, Saravia y Alvarez, 2016), Avendaño, Rueda \& Paz (2016), análizan desde el Modelo de Modernización para la Gestión ambiental de operaciones en las pequeñas y medianas empresas del sector arcilla presentes en el municipio de San José de Cúcuta y su área metropolitana (El Zulia, Villa del Rosario y Los Patios), en donde se presentan problemas de contaminación ambiental, deslizamientos, derrumbes agotamiento de cuencas hidrográficas, falta de prevención y de sistemas de control que mitiguen el impacto de la explotación, transporte y comercialización de recursos sobre el medio ambiente.

El Sector Cerámico en el departamento, muestra una tendencia al progreso y al fortalecimiento con una visión a largo plazo, estudios realizados por Cely y Bolívar (2015) buscan obtener el mayor provecho de este recurso dando valor agregado y mayor competetividad en el mercado internacional. Actualmente existen empresas legalmente constituidas, dedicadas a la producción de materiales de construcción, algunas con baja tecnología en sus procesos, principalmente en el de cocción, utilizando para ello el horno colmena, que es menos eficiente y genera contaminantes a la atmosfera a través de sus chimeneas.
Norte de Santander y principalmente el área metropolitana de Cúcuta se ha caracterizado por ser una región rica en producción de arcilla y carbón, siendo sus principales productos de comercialización y exportación. No obstante la contaminación generada por las plantas de fabricación de artículos cerámicos es una fuente de emisiones muy importantes, la elaboración de productos cerámicos está estrechamente relacionada con impactos ambientales desfavorables para el entorno, teniendo como aspecto más relevante la emisión de gases a la atmosfera provenientes de las etapas de cocción, dependiendo en gran medida del combustible que sea utilizado para la combustión en los hornos (García y Molina, 2011). El Ministerio de Ambiente, Vivienda y Desarrollo Territorial, en la resolución 909 de 2008, estableció las normas y estándares de emisión admisibles de contaminantes a la atmósfera para las industrias de fabricación de productos de cerámica refractaria, no refractaria y de arcilla (ver tabla I), así como su seguimiento y control, lo que estableció el protocolo para el Control y Vigilancia de la Contaminación Atmosférica Generada por Fuentes Fijas. $\mathrm{mg} / \mathrm{m} 3$. Estos inconvenientes reflejan un precario control de los contaminantes atmosféricos en estas empresas y sobre todo que en algunas ocasiones no se cumple con los estándares impuestos por el Ministerio, por lo tanto se plantea establecer un modelo matemático que permita de manera preventiva estimar la concentración de los contaminantes que salen de la boca de la chimenea de los hornos colmena identificados, con base en las variables que inciden en el proceso de cocción y basado sobre las ecuaciones de conservación de masa propias del proceso.

\section{Materiales y métodos}

El enfoque de la investigación es cuantitativo, de tipo explicativo correlacional, con un muestreo no probabilístico de tipo intencional, ya que se trabaja con los estudios isocinéticos de algunas fábricas que poseen las mediciones de las emisiones atmosféricas en las chimeneas (estudios isocinéticos). 
Tabla I. Estándares de emisión admisibles de contaminantes al aire para las industrias existentes de fabricación de productos de cerámica refractaria, no refractaria y de arcilla, a condiciones de referencia $\left(25^{\circ} \mathrm{C}, 760 \mathrm{~mm} \mathrm{Hg}\right)$ con oxígeno de referencia del $18 \%$.

\begin{tabular}{cccc}
\hline Conbustible & \multicolumn{4}{c}{ Estándares de emisión admisibles $\left(\mathrm{mg} / \mathrm{m}^{3}\right)$} \\
\hline & $\mathrm{MP}$ & $\mathrm{SO}_{2}$ & NOx \\
Solido & 250 & 250 & 550 \\
Liquido & 250 & 250 & 550 \\
Gaseoso & No Aplica & No Aplica & 550 \\
\hline
\end{tabular}

Fuente: Resolución 909 DE 2008, Ministerio de Ambiente, Vivienda y Desarrollo Territorial

De manera general, un estudio isocinético de una fuente de emisión fija en este caso pertinente una chimenea de horno; se efectúa con el fin de determinar el flujo másico delos contaminante a partir de la concentración de este y del flujo del gas que realiza el transporte del contaminante, estos datos son tomados directamente de la fuente de emisión a través de un muestreador de chimenea, acatando estrictamente la metodología definida por la Agencia de Protección Ambiental delos Estados Unidos, EPA.

Determinar estas concentraciones de contaminantes da un punto de partida para realizar las respectivas acciones pertinentes para mitigarla oeliminarla. Dicha razón hace que estos estudios tomen un carácter de obligatoriedad para las empresas que en sus proceso productivos generanalgún tipo de material partículado.

Por normatividad, estos controles se establecen con una periodicidad de un año o en menor tiempo si los resultados lo ameritan; cabe aclarar que dependiendo de la capacidad productiva de las empresas, se realizan números superiores de seis (6) procesos de cocción por mes, para un promedio de 72 quemas en el año, de las cuales solo un seguimiento las hace factibles o no.

Además de lo anterior, los muestreos se deben realizar con personal idóneo y equipos técnicos especializados, que no es de la región y los respectivos análisis se realizan en interior del país. Esto incrementa los costos de contratación, evitando que la periodicidad de los análisis sea reducida para incrementar los muestreos y posterior análisis respectivo.

Ante la problemática expuesta y teniendo en cuenta el contexto geográfico, la investigación se desarrolla en el municipio del Zulia, donde se concentra un número significativo de chircales y ladrilleras con baja tecnología en sus procesos, principalmente en la etapa de cocción, donde el horno más utilizado es el horno colmena, considerado uno de los menos eficientes y con altos flujos de emisiones gaseosas; y donde se debe considerar que, por concepto, tanto el diseño, la construcción y operación de los hornos colmena deberían ser igual en todos las empresas cerámicas del sector, segúnopinióndelosexpertos, existen variantes significativas entre los hornos ya que cada fabrica realiza ajustes a su conveniencia.
Este sector productivo no es ajeno a los inconvenientes de realizar los respectivos análisis, ya sea por las limitaciones en la ubicación, el costo elevado de contratación por parte de las instituciones responsables de los estudios de evaluación de emisiones atmosféricas en fuentes fijas, el temor a no cumplir con los estándares luego de desarrollar dichos análisis, teniendo el conocimiento que por parte del ministerio, esto puede conllevar a la afectación de la sostenibilidad de la empresa con sanciones, cierres parciales o permanentes.

Otra problemática que se presume tiene incidencia el sector cerámico pero sin ninguna relación confirmada públicamente, se evidencia en el informe de la Alcaldía del municipio del El Zulia, del análisis de situación en salud con determinantes sociales municipio del Zulia, Norte de Santander, año2015, en dicho informe se establece que el "respeto a la mortalidad ajustada por edad y subgrupos de causas en la población masculina, en las infecciones respiratorias agudas ocupan el primer lugar en la carga de mortalidad por dichas subcausas, evidenciándose un comportamiento variable en el período del 2005-2013, siendo el año más crítico el 2010 con una tasa de 42,56 muertes por cada 100 Hombres; En cuanto a lamortalidad por enfermedades transmisibles enmujeres, al igual que en la población masculina, las infecciones respiratorias agudas son las más representativas, cuya tasa ha presentado un comportamiento en aumento en esta población a partir del 2008, pasando deuna tasa igual a 13.24 en ese año a una tasa igual a 26.28 en el año 2013" (ver figura 1)

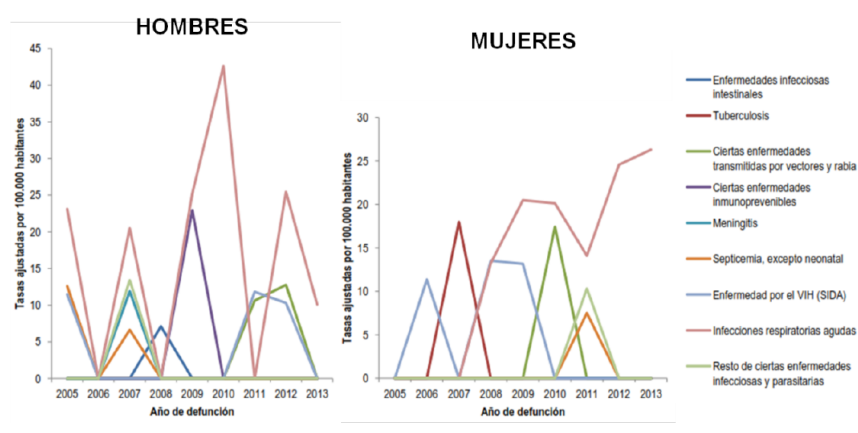

Figura 1. Tasa de mortalidad ajustada por edad para las enfermedades transmisibles en hombres y mujeres en el municipio del Zulia, 2005 -2013 Fuente:DANE - EstadísticasVitales2005 - 2013.

Conocedores de toda la problemática expuesta y tomando la resolución 909 de 2008, que exhorta en su artículo 73, a considerar métodos alternativos que faciliten los procesos de control; y por ello es interesante tener en cuenta, que se deben crear otros métodos para contribuir de manera preventiva al control de las emisiones de las chimeneas de los hornos colmena, teniendo en cuenta las diferentes variables que puedan incidir en el proceso de cocción, evitando sobre costos y facilitando un seguimiento más acertado en el tiempo, y en la cantidad de procesos de la etapa de cocción y así evitar el deterioro ambiental y del entorno. 
Con este proyecto se busca desarrollar un método alternativo de estimación de la concentración de los contaminantes de los hornos colmena, en la industria cerámica en Norte de Santander. Para lograr esta estrategia, el enfoque de la investigación es cuantitativo y de tipo exploratoria, ya que usa la recolección de datos para probar una hipótesis, con base en la medición numérica en el proceso de cocción y el análisis estadístico de los históricos de los estudios de evaluación de emisiones atmosféricas en fuentes fijas de los chircales, con el propósito de establecer patrones de comportamiento y probar la teoría del modelo matemático, estrategia de la cual no se tiene investigación relacionada alguna.

De igual manera la investigación se enmarca como no experimental cuantitativa, ya que no se manipulará deliberadamente las variables del proceso, puesto que se prestara atención a las situaciones ya existentes, ya sea en los reportes históricos de control de emisión o los procesos en su entorno natural para después analizarlos; de igual manera será un diseño transeccional exploratorio debido a que el agregado de variables son proporcionadas en situaciones reales.

También se puede considerar la investigación de tipo explicativo correlacional, pues a partir de la correlación entre variables se busca explicar el comportamiento de la variable dependiente en función de las varias variables independientes consideradas.

En el desarrollo del proyecto, tiene como población objeto a las empresas cerámicas que se encuentran ubicados en el municipio del Zulia, específicamente en la zona de la Alejandra; en relación a la muestra, se conjetura que no es obligatorio obtener una muestra característica de la población para aplicar un algoritmo matemático; y en base en las limitaciones de disponibilidad de información de estudios isocíneticos realizados, controles productivos en la etapa de cocción y el interés de participación en el proyecto se desarrolla un muestreo no probabilístico de tipo intencional en las fábricas, dadas en la tabla II.

Tabla II. Número de Hornos colmena de la empresas cerámicas colaboradoras

\begin{tabular}{cc}
\hline EMPRESA CERAMICA & NUMERO DE HORNOS COLMENA \\
\hline Merkgres & 4 \\
Solo barro S.A.S. & 2 \\
Ecobement S.A.S. & 2 \\
Arcillas de Colombia E \& M S.A.S. & 4 \\
Tejar Santa Maria Ltda. & 4 \\
Arcillas Castilla & 2
\end{tabular}

\section{Resultados y Discusión}

Dados que los análisis isocineticos que las empresas poseen son pocos, debido a lo costoso de estos estudios, donde algunas empresas poseen sólo han realizado un análisis y máximo tres, se necesita implementar la simulación de datos Metodología de Simulación Monte Carlo con el fin de tener información suficiente para modelizar.

Con los expertos se definen las variables de incidencia de los procesos productivos en la etapa de cocción, sometiendo estas a consenso con los respectivos gerentes o responsables.

En la parte del esquema estructural de los hornos se puede evidenciar que existen diferencias considerables de estos, dependiendo de las necesidades de cada empresa. Razón por la cual se deben incluir ciertas condiciones de diseño como variables.

Conociendo que el combustible utilizado en el proceso de cocción, es el carbón, es recomendable conocer las condiciones de aprobación de dicho combustible sólido fósil.

En el momento de predecir un modelo para la concentración de contaminantes se debe especificar las variables y las relaciones de causalidad.

Teniendo en cuenta los diferentes aspectos, específicamente para el modelo predictivo de través de hornos colmena del sector cerámico del municipio del Zulia, se podrían considerar como variable dependiente a la concentración de contaminantes; y como variables dependientes tendríamos: altura de la chimenea, volumen del horno, el balance de masa, la cantidad de carbón y la masa de material a quemar. Como variable interviniente en este caso se toma Porcentaje de azufre en el carbón. Donde la relación se expresa mediante la figura 2

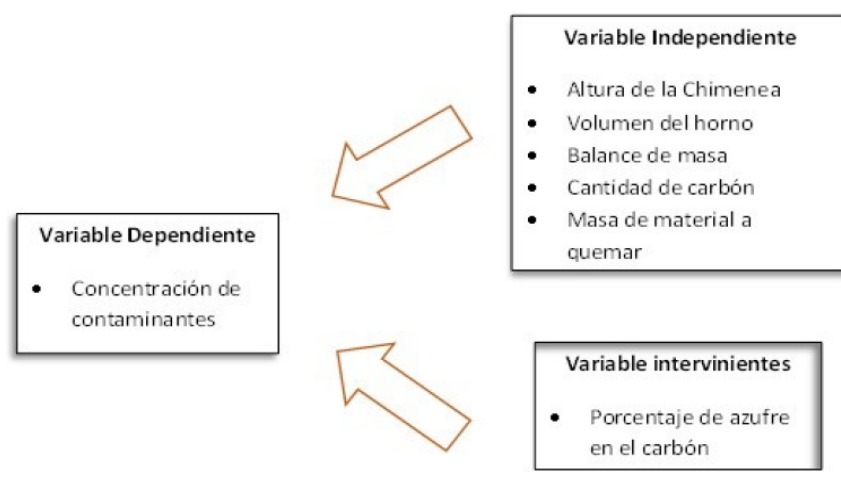

Figura 2. Sistema de variables en el Modelo predictivo de Concentración de Contaminantes Fuente: Autor 


\section{Conclusiones}

Avendaño, Rueda \& Paz (2016), en su artículo La gestión ambiental en las pymes del sector arcilla en Cúcuta y su área metropolitana con el diagnóstico realizado identificaron que las acciones encaminadas a la gestión ambiental las realizan bajo intereses legales, sin lograr un verdadero compromiso empresarial con el bien social, enmarcándose en la mayoría de las gestiones a criterios de origen legal.

Lo que evidencia la necesidad de diseñar e implementar acciones, planes y programas tanto preventivos como correctivos en la gestión ambiental, en aspectos como el ruido, fortalecimiento de los departamentos de gestión ambiental, el tratamiento adecuado de residuos sólidos y la mitigación de emisiones atmosféricas.

Una vez propuesto el modelo predictivo se puede estimar el valor de la concentración de contaminantes de cada uno de los hornos con el sólo conocimiento de las variables independientes que considera el modelo.

Esta información podrá ser el punto de partida para el control por parte de los organismos del Estado al exigirle a las empresas nuevas metas en la concentración de contaminantes y por supuesto la modificación de variables independientes como la altura y volumen de la chimenea para la construcción de nuevos hornos de carácter más ecológico.

Por otra parte, a lo interno de cada empresa, al conocer su realidad podrán revisar sus líneas y metas de producción y establecer políticas más pertinentes con los lineamientos nacionales e internacionales en conservación delambiente.

También se pretende generar una herramienta que dote al personal involucrado en la toma de decisiones de las diferentes empresas a poder proyectar en el tiempo sus emisiones atmosféricas, y realizar ajustes a las distintas variables en el modelo encaminadas hacia la mitigación de los mismos; situación que posteriormente se puede ajustar a la realidad de sus procesos productivos.

En el desarrollo de la fase exploratoria del modelo se encontraron ciertas anomalías, tales como: las variables capacidad y producción, son prácticamente idénticas lo que conllevan a una alta correlación y en consecuencia y una multicolinealidad no deseada entre las variables, por lo que la sugerencia es omitir una de éstas y se considera la variable Producción en Lugar de Capacidad y la variable Porcentaje de Azufre correlaciona en forma inversa con las mayoría de las variables y su saturación no es clara por lo que teóricamente se sostiene como una variable latente del indicador $\mathrm{SO} 2$.

\section{Referencias}

Alcaldía del municipio del El Zulia(2016). Análisis de Situación en Salud con Determinantes Sociales municipio del Zulia, Norte de Santander. Disponible en: http://salasituacionalidsnds.weebly.com/uploads/1/0/7/1/1 0714324/asis-departamental-2016-nortedesantander.pdf.

Avendaño, W., Rueda, G. \& Pas, L. (2016). La gestión ambiental en las pymes del sector arcilla en Cúcuta y su área metropolitana. Revista Finanzas y Política Económica, 8(1), 123-155.

Cauas, D. (2015). Definición de las variables, enfoque y tipo de investigación. Biblioteca electrónica de la Universidad Nacional Abierta ya Distancia. Recuperado de: http://datateca. unad. edu. co/contenidos/210115/Documento_reconociemiento_Unidad_N o_2.pdf\#page, 4.

Cely-Illera, L., \& Bolívar-León, R. (2015). Materia prima para la industria cerámica de Norte de Santander. II. Evaluación del comportamiento térmico y su incidencia en las propiedades tecnológicas. Respuestas, 20(1), 84-94. https://doi.org/10.22463/0122820X.260

García, J. I. D., \& Molina, J. S. (2011). Introducción a los hornos utilizados en la industria cerámica tradicional. Editorial Universidad Francisco de Paula Santander.

Díaz, L. (2013). Trapitos al Arte, Mundo Fesc, 3(5), 52-56.

García S. J. P.\&. Maheut J. (2015). Modelado y Resolución de Problemas de Organización Industrial mediante Programación Matemática Lineal

Krastek, R. et al. (2012). Formulación de un modelo matemático para optimizar el tiempo de producción en una planta extrusora de tubos.

Ministerio de Ambiente, Vivienda y Desarrollo Territorial. Resolución 909 de 2008.Por la cual se establecen las normas y estándares de emisión admisibles de conta minantes a la atmósfera por fuentes fijas y se dictan otras disposiciones.

Sánchez-Molina, J., Sarabia-Guarin, A., \& Alvarez-Rozo, D. (2016). Evaluación de materias primas utilizadas en la fabricación de baldosas de gres en el sector cerámico de Norte de Santander (Colombia). Respuestas, 21(2), 48 -56. https://doi.org/10.22463/0122820X.776

Sarabia-Guarin, A., Sánchez-Molina, J., \& Leyva-Díaz, J. (2017). Uso de nutrientes tecnológicos como materia prima en la fabricación de materiales de construcción en el paradigma de la economía circular. Respuestas, 22(1), 6 16. https://doi.org/10.22463/0122820X.815 